\title{
Hunting Gravitational Wave Mergers with Cosmic Surveys
}

\section{INTRODUCTION}

Nearly 60 gravitational wave $(\mathrm{GW})$ events from merging compact object binaries (binary black holes, binary neutron stars and neutron star-black hole systems) have been detected by the LIGO and Virgo gravitational wave detectors. The first gravitational wave multi-messenger detection of GW170817, associated to the kilonova

AT2017gfo which was discovered by the Dark Energy Survey (DES) amongst other teams in 2017, has also been the last GW event with an associated counterpart.

The DES-GW team is now hunting down new associations of GW detections with optical emission visible with the Dark Energy Camera, a massive camera built at Fermilab for the DES, and now mounted on the Blanco telescope in Chile.

\section{OBJECTIVES}

- Use existing DES data to search for the optical emission expected from binary neutron stars or binary black holes emitting gravitational waves in active galactic nuclei (AGN) disks, which are a good candidate for hosting massive stellar black hole mergers.

- Fit flare models to light curve data

- Work towards finding a constraint on $\lambda$ : the fraction of Binary Black Holes that occur in AGNs

- Handle analyses of big data in astrophysics

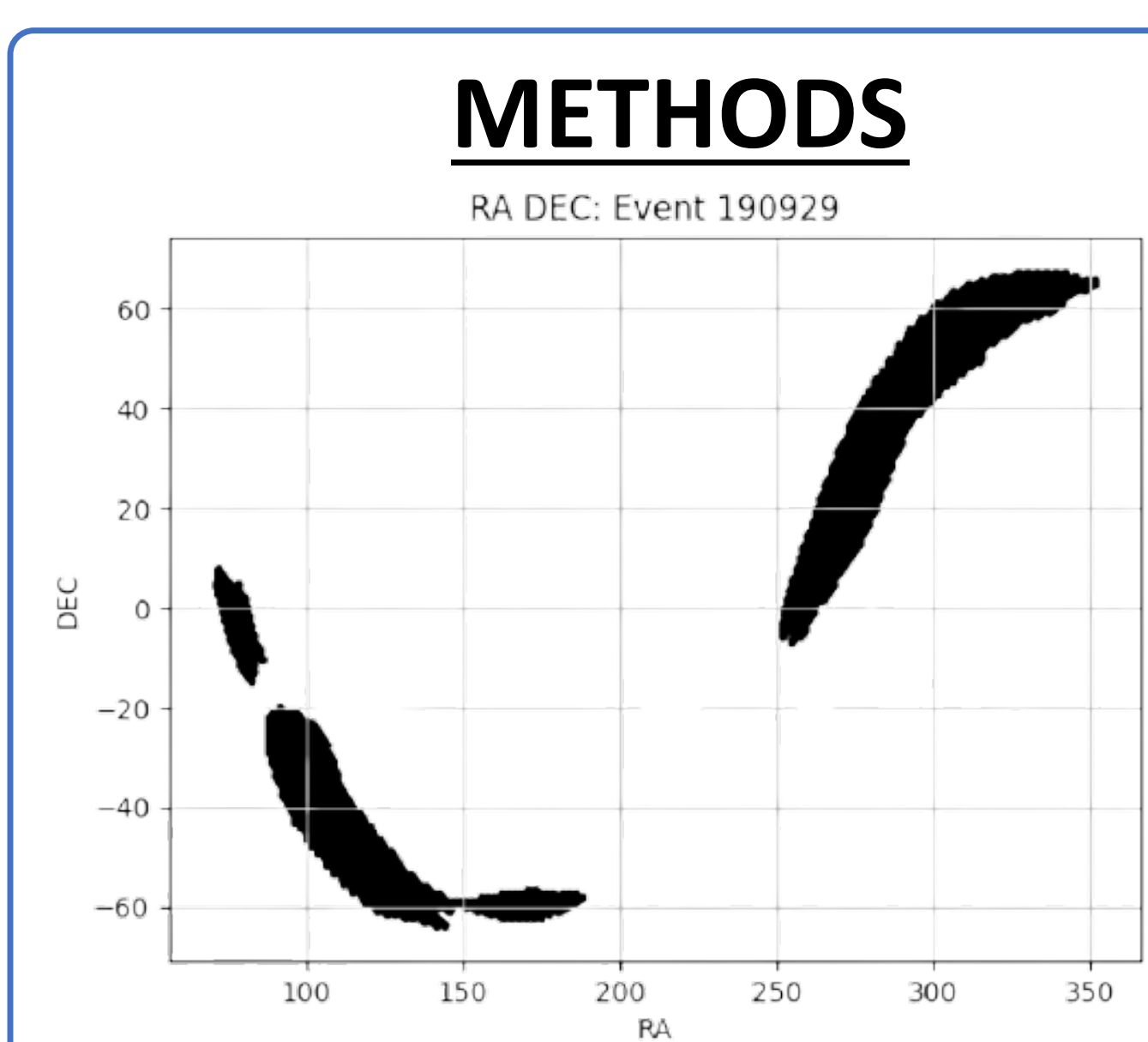

Figure 1. Skymap showing the $90 \%$ probability location range for $\mathrm{GW}$ event 190929.

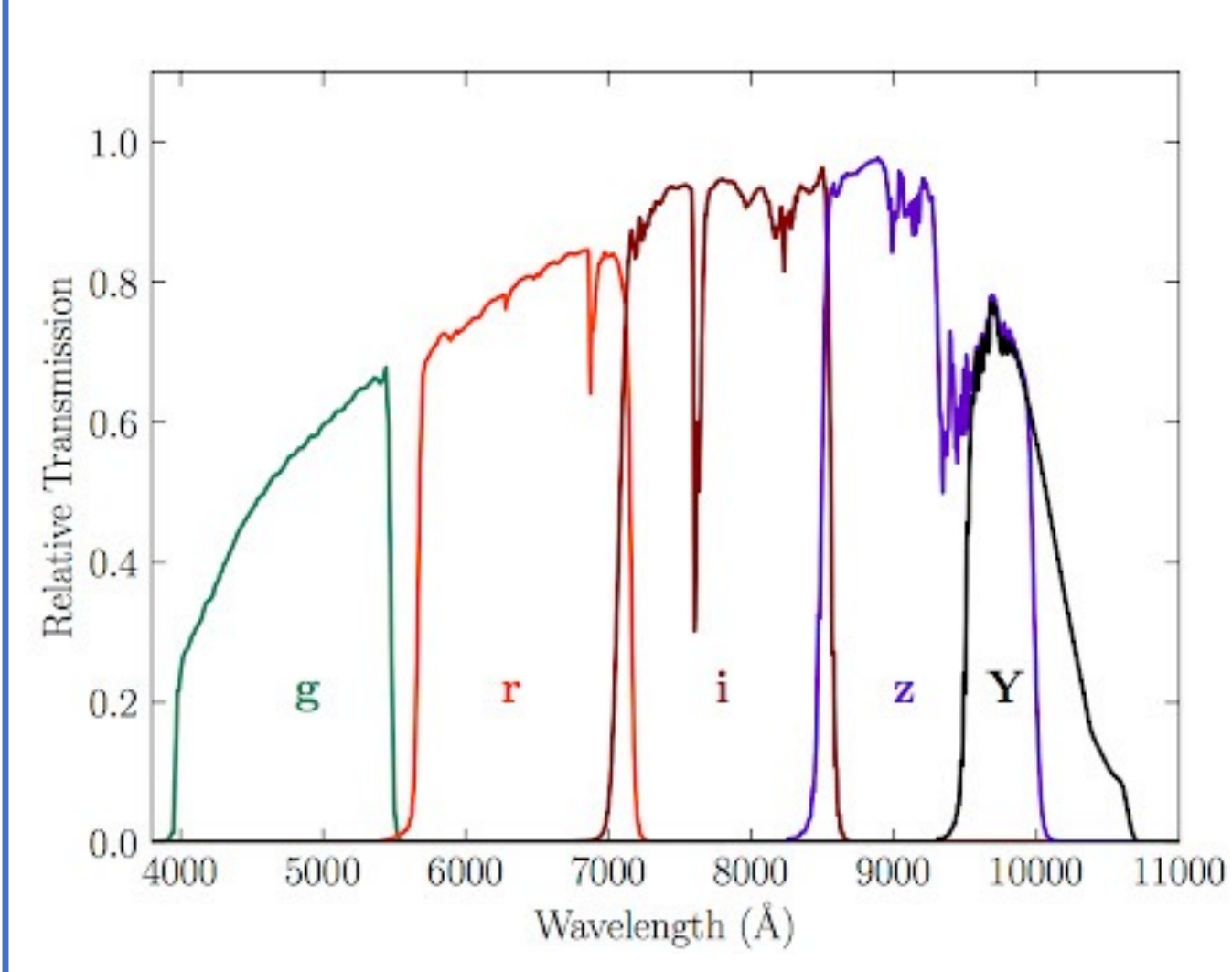

Figure 2. DES DR1 Standard Bandpasses for the DECam g, r, i, z, and y filters. Light curves are separated based on these filters.

This manuscript has been authored by Fermi Research Alliance, LLC under Contract No. DE-AC02-07CH11359 with the U.S. Office of High Bnagy Physict

\section{RESULTS}
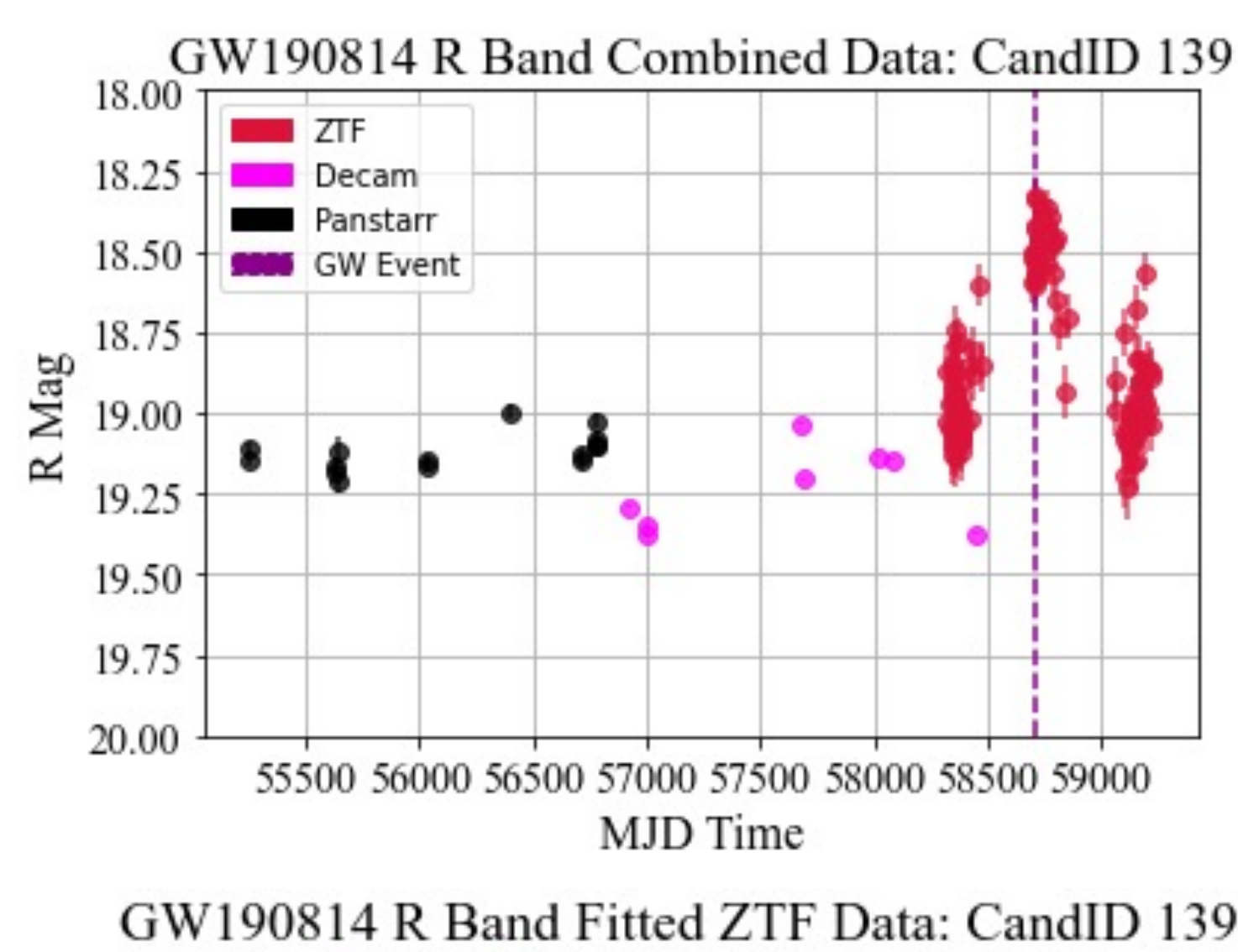

Figure 4. Curve with a Gaussian rise/exponential

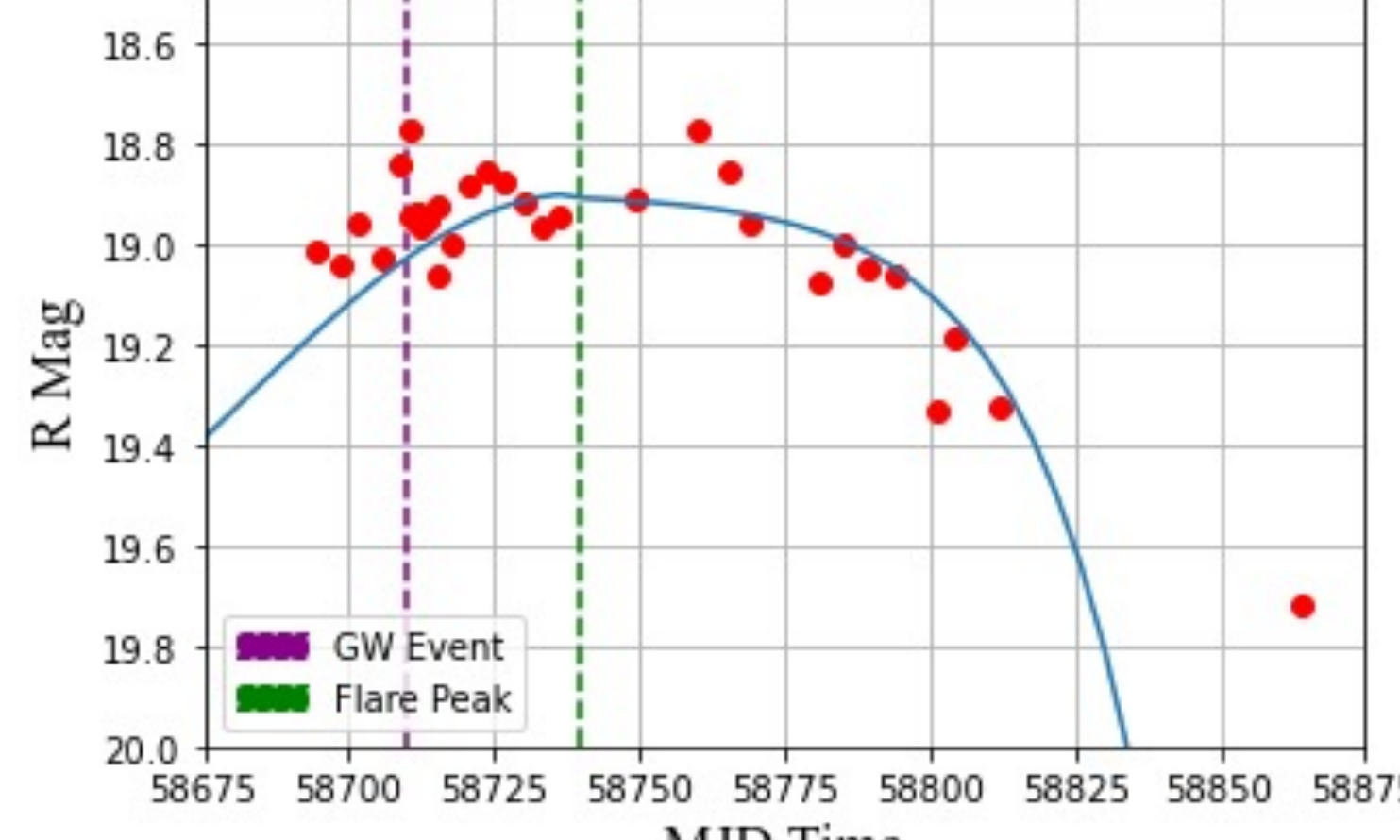
decline fitted to $\mathrm{R}$ Band flare data. Fit is optimized for the locus of the GW Event, up to \pm 200 MJD away

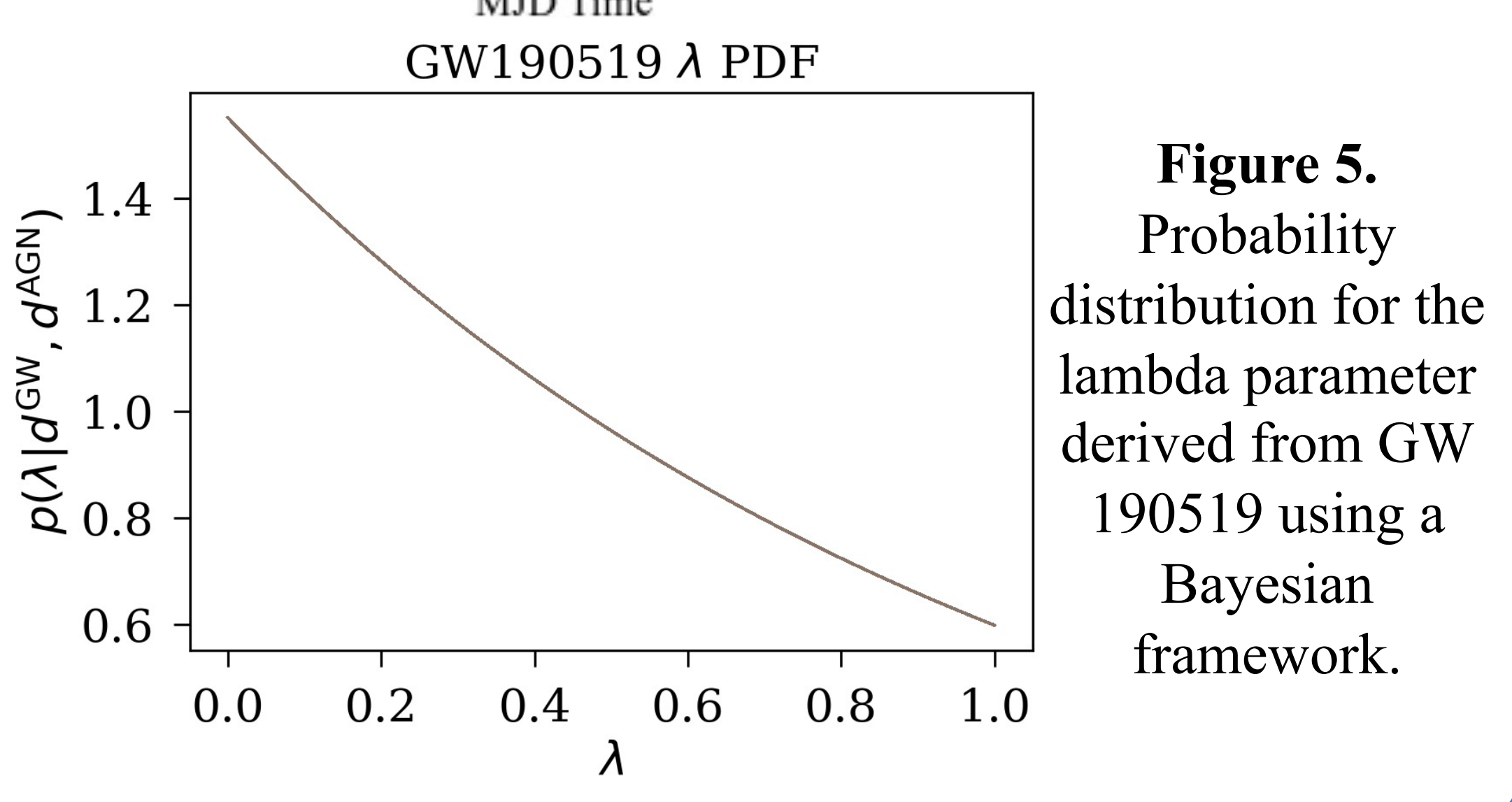

\section{CONCLUSIONS}

- Specific flare for GW190814 (candID 139) not consistent with gravitational wave event

- Analysis ongoing for currently available GW data

- Gaussian rise/exponential decline fit found for general locus of the GW event

- Lambda upper 2-sigma: 0.929

\section{FUTURE WORK}

New discoveries of GW counterparts using DECam will help shed light on some of the unknowns related to these systems, including their origin and the production of the heaviest elements in the Universe, and they will enable measurements of the expansion of the Universe. Future work will involve searching for flares like candID 139 and exploring the constraints on $\lambda$ using future events.

\section{REFERENCES}

[1] Graham et. al 2020, Phys. Rev. Lett. 124, 251102

[2] Garcia et. al. arXiv:2007.00050v1 [astroph.HE]

[3] McKernan et. al. 2019 ApJ, 884, L50

[4] Palmese et. al. The Astrophysical Journal Letters, 2020, Volume 900, Number 2

[5] Palmese et. al. The Astrophysical Journal Letters, Volume 914, Issue 2, id.L34 (2021) 